\title{
Differential circular RNA expression profiles in umbilical cord blood exosomes from preeclampsia patients
}

Minkai Cao ${ }^{1 \dagger}$, Juan Wen ${ }^{2 \dagger}$, Chaozhi Bu ${ }^{3}$, Chunyan $\mathrm{Li}^{4}$, Yu Linn ${ }^{4}$, Hong Zhang ${ }^{1}$, Yanfang Gu', Zhonghua Shi ${ }^{4}$, Yan Zhang ${ }^{1 *}$, Wei Long ${ }^{4^{*}}$ and Le Zhang ${ }^{5^{*}}$ (D)

\begin{abstract}
Background: Exosomal circular RNAs (circRNAs) are emerging as important regulators of physiological development and disease pathogenesis. However, the roles of exosomal circRNAs from umbilical cord blood in preeclampsia (PE) occurrence remains poorly understood.

Methods: We used microarray technology to establish the differential circRNA expression profiles in umbilical cord blood exosomes from PE patients compared with normal controls. Bioinformatics analysis was conducted to further predict the potential effects of the differentially expressed circRNAs and their interactions with miRNAs.

Results: According to the microarray data, we identified 143 significantly up-regulated circRNAs and 161 significantly down-regulated circRNAs in umbilical cord blood exosomes of PE patients compared with controls. Gene Ontology (GO) and Kyoto Encyclopedia of Genes and Genomes (KEGG) biological pathway analyses showed that circRNA parental genes involved in the regulation of metabolic process, trophoblast growth and invasion were significantly enriched, which play important roles in PE development. Moreover, pathway network was constructed to reveal the key pathways in PE, such as PI3K-Akt signaling pathway. Further circRNA/miRNA interactions analysis demonstrated that most exosomal circRNAs had miRNA binding sites, and some miRNAs were associated with PE.
\end{abstract}

Conclusions: Our results highlight the importance of exosomal circRNAs in the pathogenesis of PE and lay a foundation for extensive studies on the role of exosomal circRNAs in PE development.

Keywords: Umbilical cord blood, Exosomes, Circular RNA, Preeclampsia

\footnotetext{
* Correspondence: fuyou2007@126.com; wlong@njmu.edu.cn; zhle@njmu.edu.cn

${ }^{\dagger}$ Minkai Cao and Juan Wen contributed equally to this work.

${ }^{1}$ Department of Obstetrics, The Affiliated Wuxi Maternity and Child Health

Care Hospital of Nanjing Medical University, Wuxi 214002, China

${ }^{4}$ Department of Obstetrics, Women's Hospital of Nanjing Medical University,

Nanjing Maternity and Child Health Care Hospital, Nanjing 210004, China

${ }^{5}$ Department of Neonatology, The Affiliated Wuxi Children's Hospital of

Nanjing Medical University, Wuxi 214023, China

Full list of author information is available at the end of the article
}

(C) The Author(s). 2021 Open Access This article is licensed under a Creative Commons Attribution 4.0 International License, which permits use, sharing, adaptation, distribution and reproduction in any medium or format, as long as you give appropriate credit to the original author(s) and the source, provide a link to the Creative Commons licence, and indicate if changes were made. The images or other third party material in this article are included in the article's Creative Commons licence, unless indicated otherwise in a credit line to the material. If material is not included in the article's Creative Commons licence and your intended use is not permitted by statutory regulation or exceeds the permitted use, you will need to obtain permission directly from the copyright holder. To view a copy of this licence, visit http://creativecommons.org/licenses/by/4.0/ The Creative Commons Public Domain Dedication waiver (http://creativecommons.org/publicdomain/zero/1.0/) applies to the data made available in this article, unless otherwise stated in a credit line to the data. 


\section{Background}

Preeclampsia (PE) is a gestation-specific syndrome that affects up to $5-7 \%$ of pregnancies, characterized by elevated blood pressure and proteinuria after 20 weeks of pregnancy $[1,2]$. This multisystem pregnancy disorder is often accompanied by headache, nausea, vomiting, upper abdominal discomfort and other symptoms, and is a leading cause of maternal and neonatal morbidity and mortality worldwide [3]. Due to the multifactorial nature of the disorder, the exact etiology of PE remains largely unknown, and there is currently no effective treatment for $\mathrm{PE}$ other than termination of pregnancy. It is reported that $\mathrm{PE}$ and its related diseases are responsible for nearly $40 \%$ of premature births delivered before 35 weeks of gestation [4]. After giving birth, although most women return to normal blood pressure levels, PE reflects an elevated long-term risk of cardiovascular diseases in both the mother and the child [5-7].

Exosomes have become key mediators of local and systemic intercellular communication by regulating different biological processes between cells $[8,9]$. Many studies have reported that exosomes play an important role in the regulation of pregnancy complications such as preeclampsia and gestational diabetes $[10,11]$. Specific exosomes derived from placenta can be detected in maternal blood as early as the 6th week of gestation, and concentrations of placentaderived exosomes increase as pregnancy progresses [12]. In addition, exosomes isolated from maternal blood are biologically active in vitro and can enter target cells through endocytosis [13]. Circular RNA (circRNA) is a novel member of endogenous noncoding RNAs, which is widely distributed and has a variety of cellular functions. Recently, circRNAs have been identified for their enrichment and stability in exosomes $[14,15]$. More and more studies have shown that exosomal circRNAs are involved in the processes of cell growth, angiogenesis, epithelial mesenchymal transition and targeted therapy $[16,17]$. And accumulating evidences suggested that it is possible to identify functional and/or structural differences in umbilical cord blood with risk of PE [18-20]. Therefore, we speculated that exosomal circRNA in umbilical cord blood might play an important role in the regulation of PE development as a new placental derived factor. However, the role of exosomal circRNAs from umbilical cord blood in PE development remains unclear. In this study, we used microarray technology to construct a comparative exosomal circRNA profiling of umbilical cord blood between PE patients and controls, aiming to lay a foundation for further research on the role of exosomal circRNAs in PE development.

\section{Methods}

\section{Patients and sample collection}

All participants and clinical information were collected at the Nanjing Maternity and Child Health Care
Hospital and Wuxi Maternity and Child Health Care Hospital from September 2019 to February 2020. PE was defined as new-onset hypertension (blood pressure $\geq$ $140 / 90 \mathrm{mmHg}$ on two separate occasions at least $6 \mathrm{~h}$ apart or blood pressure $\geq 160 / 110 \mathrm{mmHg}$ ) and proteinuria (> $300 \mathrm{mg} / 24 \mathrm{~h}$ ) after 20 weeks of gestation in previously normotensive women. The pregnant women without PE were included as controls. The controls were matched to PE cases for maternal age. In order to reduce selection bias with different PE severity, pregnant women with gestational age greater than 34 weeks were included in this study. At last, 46 umbilical cord blood samples were collected from the umbilical vein immediately after delivery of fetus during cesarean section (23 PE patients and 23 controls) according to the standard operating procedure. All participants were divided into two sets, 6 participants (3 PE patients and 3 controls) as pilot sample for microarray screening and 40 participants (20 PE patients and 20 controls) for validation. The basic information of the 46 subjects was shown in Table S1.

\section{Purification and analysis of exosomes}

Exosomes were extracted from the umbilical cord blood. In brief, umbilical cord blood was centrifuged at $3000 \mathrm{~g}$ for $15 \mathrm{~min}$ at $4{ }^{\circ} \mathrm{C}$. Then supernatants were centrifuged at $12,000 \mathrm{~g}$ for $30 \mathrm{~min}$ at $4{ }^{\circ} \mathrm{C}$. The supernatants were then filtered by a $0.45 \mu \mathrm{m}$ polyvinylidene fluoride membrane, and finally isolated by ultracentrifugation at 100 , $000 \mathrm{~g}$ for $180 \mathrm{~min}$ at $4{ }^{\circ} \mathrm{C}$. The exosome particles were resuspended in lysis buffer or PBS. The resulting exosomes were then analyzed using Nanosight Nano ZS device (Malvern Instruments, UK).

\section{Exosomal RNA extraction and microarray analysis}

Total exosomal RNA was extracted using exoRNeasy Serum/Plasma Maxi Kit (QIAGEN, Germany) according to the manufacturer's instructions and checked for RNA integrity by an Agilent Bioanalyzer 2100 (Agilent technologies, USA). Total RNA was amplified and labeled by Low Input Quick Amp Labeling Kit (Agilent technologies, USA), following the manufacturer's instructions. Labeled cRNA was purified by RNeasy mini kit (QIAG EN, Germany). Each slide was hybridized with $1.65 \mu \mathrm{g}$ Cy3-labeled cRNA using Gene Expression Hybridization Kit (Agilent technologies, USA) in Hybridization Oven (Agilent technologies, USA), according to the manufacturer's instructions. After $17 \mathrm{~h}$ hybridization, slides were washed in staining dishes (Thermo Shandon, USA) with Gene Expression Wash Buffer Kit (Agilent technologies, USA), followed the manufacturer's instructions. Slides were scanned by Agilent Microarray Scanner (Agilent technologies, USA) with default settings. Data were extracted with Feature Extraction software 12.0 (Agilent 
technologies, USA). Raw data were normalized by Quantile algorithm, limma packages in $\mathrm{R}$.

\section{Quantitative real-time PCR (qPCR)}

After the total exosomal RNA was extracted and RNA integrity was checked according to the above method, RNA quantity and quality were measured with a Nano Drop ND-1000 spectrophotometer (Thermo, USA). Then, the RNA was digested using RNase R (Epicenter Biotechnologies, USA) and purified. Complementary DNA was acquired from reverse transcription of $500 \mathrm{ng}$ RNA using PrimeScript RT reagent with gDNA Eraser (TaKaRa, Japan). Primer-BLAST was applied to design the specific divergent primers, which were used to amplify the circular transcripts by head-to-tail splicing. All primers were synthesized by Realgene (Nanjing, China). After the optimal annealing temperatures were determined, qPCR was performed on the Life Tech-ViiA7 system (Applied Biosystems, USA) using PowerUP SYBR Green Master Mix (Applied Biosystems, USA) to measure the relative expression levels of circRNAs. To reduce the experimental random error, samples were loaded in triplicate and each well was treated identically. Glyceraldehyde phosphate dehydrogenase (GAPDH) was used as internal control, and the relative expression levels of circRNAs were calculated with $2^{-\Delta \Delta C t}$ method. Moreover, to guarantee the accuracy of the results, all data are represented as the means \pm standard deviation (SD) of three independent experiments.

\section{Functional enrichment analyses}

DAVID Bioinformatics Resources 6.8 was used to analyze the parental gene functions of the differentially expressed circRNAs. Gene Ontology (GO) analysis of the parental genes was conducted based on biological processes, cellular components and molecular functions (https://www.geneontology.org/). And Kyoto Encyclopedia of Genes and Genomes (KEGG, https://www.genome.jp/ kegg) was used to analyze the related biological pathways. The $-\log (P$-value $)$ was used as the enrichment score indicating the significance of correlation.

\section{Annotation of circRNA/miRNA interactions}

The miRanda (https://www.microrna.org/microrna/ home.do/) was used to predict circRNA/miRNA interactions. Using the database, we searched for miRNA response elements (MREs) on circRNAs and selected miRNAs according to the seed matching sequences.

\section{Statistical analyses}

Statistical analyses were conducted by SPSS 18.0 and GraphPad prism 5.0. All data are shown as mean \pm SD. The student's $t$-test was used to evaluate the differences between the experimental groups. Differences with $P<0.05$ were considered statistically significant.

\section{Results \\ CircRNA expression profiling in umbilical cord blood exosomes from PE patients compared with controls}

We firstly performed a primary analysis of the microarray data to investigate the basic characteristics of all the circRNAs in umbilical cord blood exosomes. We assessed a total of 88,371 circRNAs, which are known from circBase (https://circrna.org/). Through hierarchical cluster analysis, we found that the levels of circRNA expression in umbilical cord blood exosomes were distinguishable between PE patients and the control group (Fig. 1a). Based on the screening criteria of fold change (FC) $\geq 2$ and $P<$ 0.05 , a total of 304 circRNAs were screened as differentially expressed circRNAs. In the PE patients, 143 circRNAs were upregulated and 161 circRNAs were downregulated (Fig. 1b). Scatter plot and volcano plot were used to show circRNAs differentially expressed and circRNAs significantly differentially expressed, respectively (Fig. 1c and Fig. 1d). Table 1 lists the top 20 up and downregulated circRNAs. The results showed that the expression of circRNAs in cord blood exosomes was different between PE patients and the control group. Furthermore, Fig. 1e describes the chromosomal distribution of differentially expressed circRNAs and Fig. If shows that the lengths of the differential circRNAs were mostly shorter than $2000 \mathrm{bp}$.

\section{Validation of differentially expressed circRNA by qPCR}

Based on relatively high abundance, $\mathrm{FC} \geq 4, P<0.01$, and their host genes, we selected 12 candidate circRNAs to validate their expression in umbilical cord blood exosomes from additional $20 \mathrm{PE}$ patients and 20 controls, including 6 up-regulated circRNAs (circ_0081910, circ 0037771, circ_0077260, circ_0043597, circ_0042232 and circ_0090100) and 6 down-regulated circRNAs (circ 0029899, circ_0066266, circ_0036082, circ_0076206, circ_0079385 and circ_0011389). In parallel with the microarray data, qPCR results showed that the expression of circ_0077260 and circ_0090100 were increased, and the expression of circ_0076206 were decreased in PE patients (Fig. 2).

\section{GO and KEGG pathway analysis of the circRNA parental genes}

The biological functions of these differentially expressed circRNAs were detected by GO and KEGG pathway analysis. In GO analysis, the number of parental genes corresponding to $\mathrm{GO}$ entries was determined, and the -log $(P$-value) was taken as enrichment score. For biological process, the terms that contained the most genes were cellular process $(\mathrm{GO}: 0009987$, count $=180)$ and single- 
A

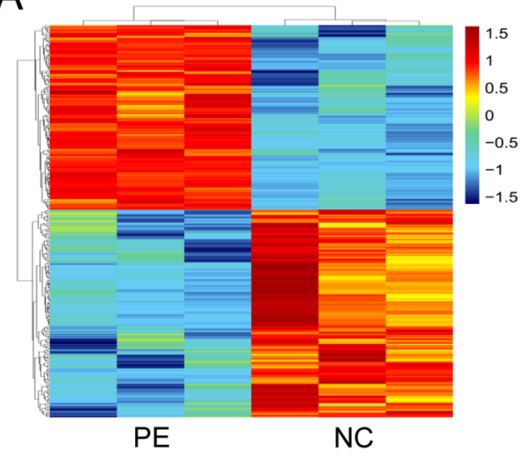

B

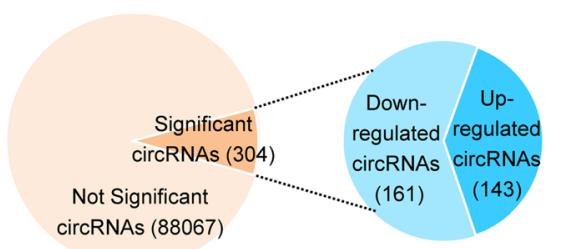

$\mathrm{D}$

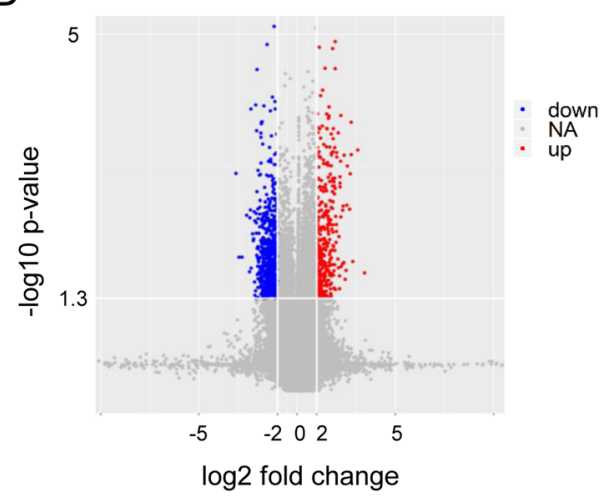

$\mathrm{F}$
$E$

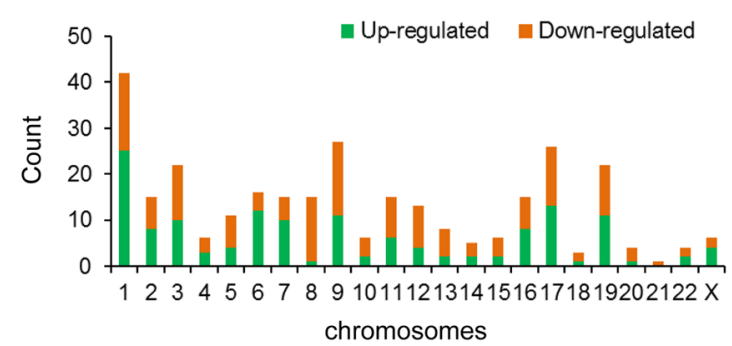

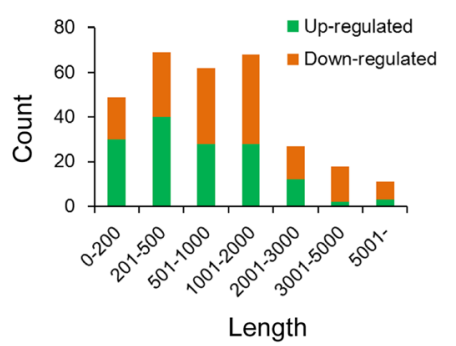

Fig. 1 CircRNA expression profiling in umbilical cord blood exosomes from PE patients compared with controls. a: Clustered heat map analysis of differentially expressed circRNAs; $\mathbf{b}$ : The total circRNAs detected by microarray and differentially expressed circRNAs between two groups; $\mathbf{c}$ Scatter plots of circRNAs signal values; d: Volcano plots visualizing the differentially expressed circRNAs; e: Chromosome distributions of the differential circRNAs; $\mathbf{f}$ : Length distributions of the differential circRNAs

organism process $(\mathrm{GO}: 0044699$, count $=163)$, and the most significantly enriched term was cell-substrate junction assembly (GO:0007044, $P=1.73 \mathrm{E}-05$ ). For cellular component, the terms that contained the most genes were cell (GO:0005623, count $=189$ ) and cell part (GO:0044464, count $=182)$, and the most significantly enriched term was focal adhesion (GO:0005925, $P=2.30 \mathrm{E}-05)$. And for molecular function, the terms that contained the most genes were binding (GO:0005488, count $=179$ ) and catalytic activity (GO:0003824, count $=114)$, and the most significantly enriched term was catalytic activity (GO:0003824, $P=$
8.90E-06) (Fig. 3). All mRNAs annotated involved in these GO terms were listed in Table $\mathrm{S} 2$.

Furthermore, the KEGG results indicated that differentially expressed circRNAs were involved in ECM-receptor interaction (hsa04512), focal adhesion (hsa04510), glycosaminoglycan degradation (hsa00531), fatty acid metabolism (hsa01212), fatty acid biosynthesis (hsa00061) and Notch signaling pathway (hsa04330) (Fig. 4). All mRNAs annotated involved in these pathways were listed in Table S3. Then, we used these pathways to construct a pathway network to investigate the key pathways in PE. As is 


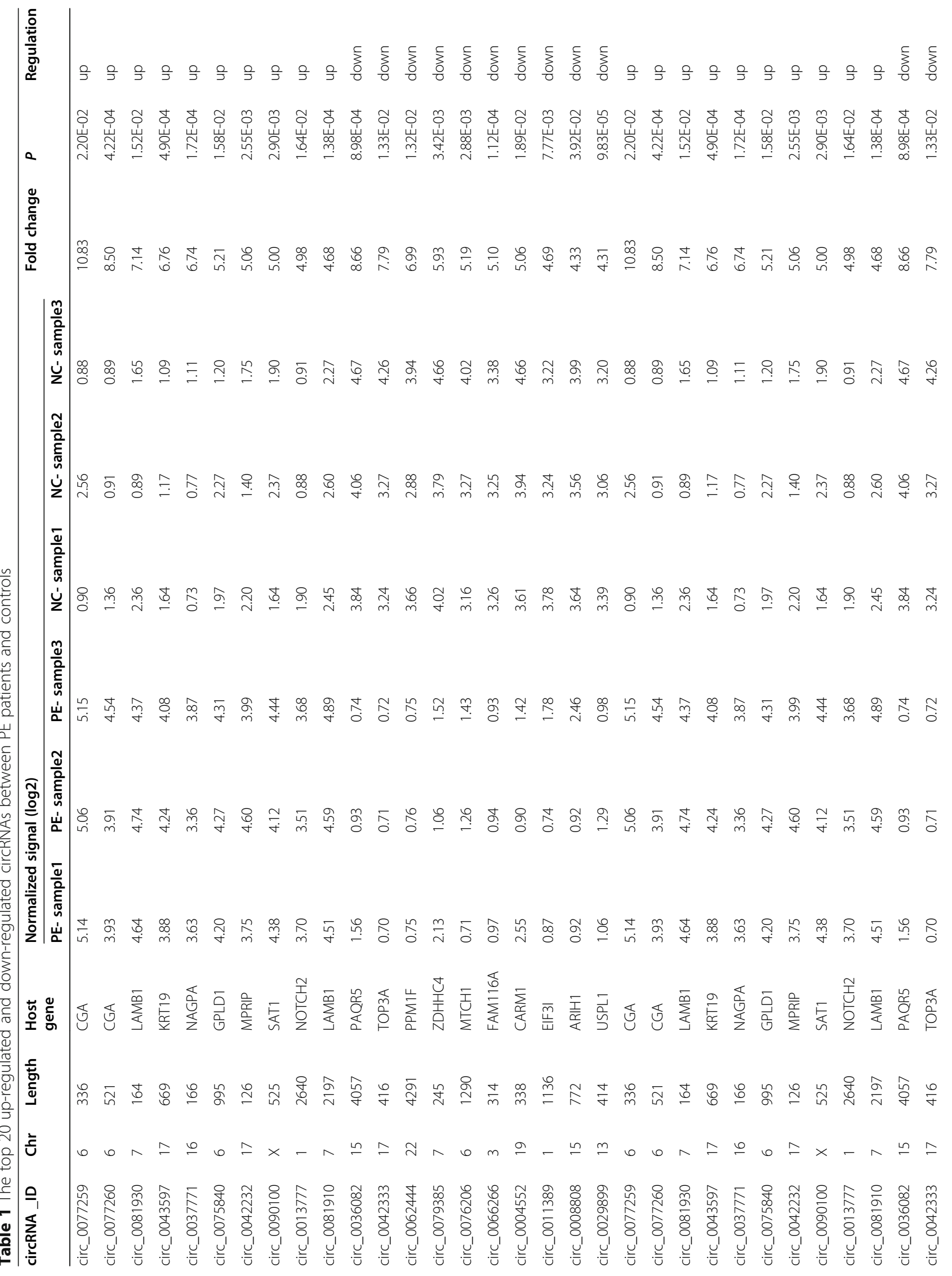




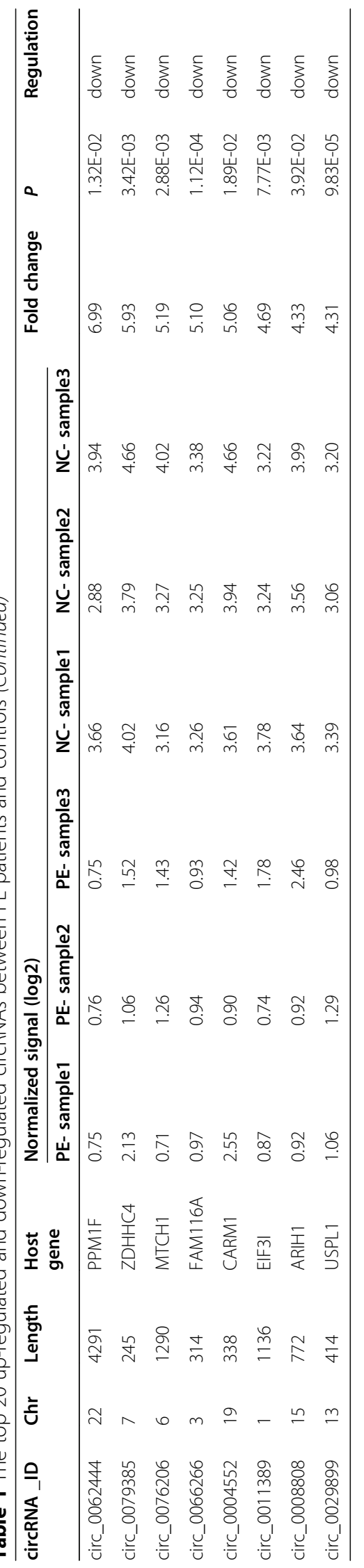




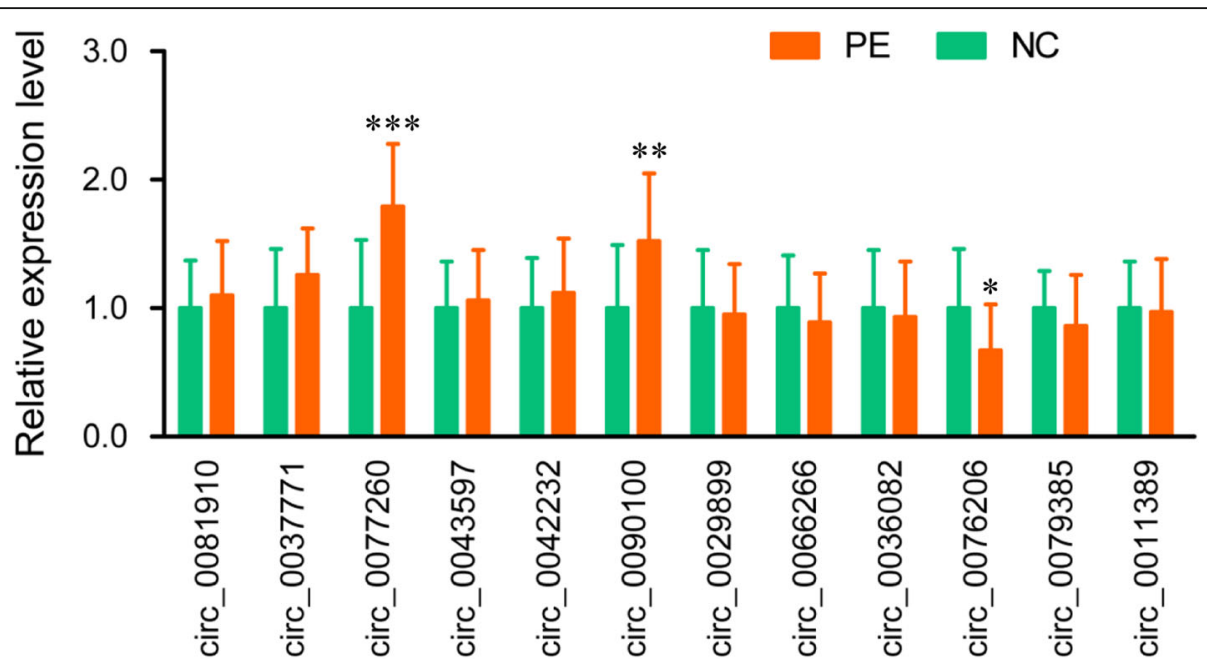

Fig. 2 Validation of differentially expressed circRNA by $q P C R$. ${ }^{*} P<0.05$, ${ }^{*} P<0.01$ and ${ }^{* *} P<0.001$

\section{Top 30 of GO Enrichment}

trabecula morphogenesis regulation of glucose metabolic process regulation of generation of precursor metabolites and energy regulation of focal adhesion assembly regulation of erythrocyte differentiation regulation of dendrite morphogenesis regulation of dendrite development regulation of ATP metabolic process pyruvate metabolic process protein serine/threonine phosphatase activity positive regulation of protein binding positive regulation of endocytosis phosphoprotein binding nucleotide phosphorylation hydrogen peroxide metabolic process glycosaminoglycan catabolic process glycolytic process focal adhesion assembly fatty-acyl-CoA biosynthetic process extrinsic component of cytoplasmic side of plasma membrane extracellular matrix structural constituent erythrocyte homeostasis erythrocyte differentiation cell-substrate junction assembly basement membrane azurophil granule lumen ATP generation from ADP ADP metabolic process adherens junction assembly acyl-CoA metabolic process -

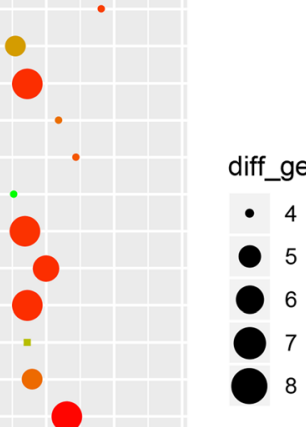

(

-

-

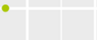
pvalue

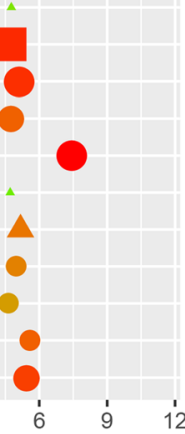

enrich factor

Fig. $3 \mathrm{GO}$ analysis of the differential circRNA parental genes 


\section{Top 30 of Pathway Enrichment}

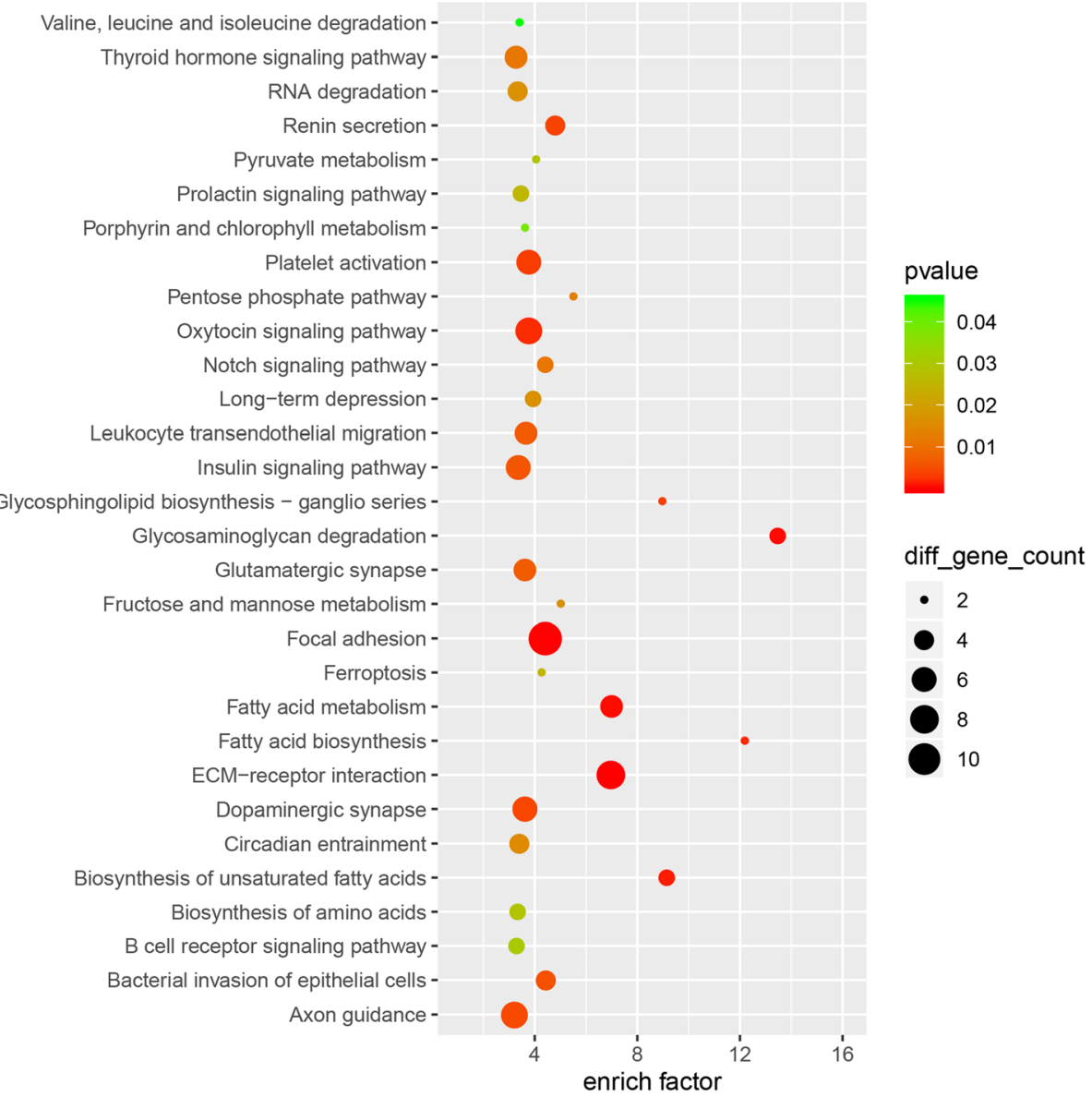

Fig. 4 KEGG pathway analysis of the differential circRNA parental genes

shown in Fig. 5, the exchanges with these pathways largely depended on the existence of PI3K-Akt signaling pathway.

\section{Prediction of circRNA/microRNA interactions}

Studies have shown that RNAs regulate each other with miRNA response elements (MREs) and this mechanism is called the hypothesis of "competing endogenous RNA (ceRNA)" [21]. CircRNAs could act as ceRNA molecules or effective miRNA sponges to regulate the expression, transcription, and protein synthesis of miRNA-targeted genes [21]. The miRanda was used to predict the interactions between the differentially expressed circRNAs and miRNAs based on MREs. Based on the criteria of $\max$ score $\geq 140$ and $\max$ energy $\leq-20$, 2226 miRNAs were found to pair with 302 differentially expressed circRNAs (Table S4); the lower the maximum energy, the more significant the correlation. The results suggest that circRNAs may be involved in the pathogenesis of $\mathrm{PE}$ through interactions with $\mathrm{PE}$ incidence-related miRNAs.

\section{Discussion}

Exosomal circRNAs have become the focus of research in recent years because of their distant regulatory potency [15]. In this study, the differential expression profile of circRNA in umbilical cord blood exosomes of PE patients was constructed for the first time, providing a basis for subsequent studies on the relationship between exosomal circRNA and PE development. Based on the microarray data, we identified 304 differentially expressed circRNAs in umbilical cord blood exosomes of PE patients when compared with normal controls, including 143 upregulated circRNAs and 161 downregulated circRNAs, which indicated that the expression pattern of exosomal circRNAs in PE patients was different from that in controls.

In subsequent validation experiments, the expression of exosomal circ_0077260 and circ_0090100 were significantly increased, and the expression of exosomal circ_0076206 were significantly decreased in PE samples. Growing evidence has shown that circRNAs can regulate parental gene expression through diverse mechanisms, 


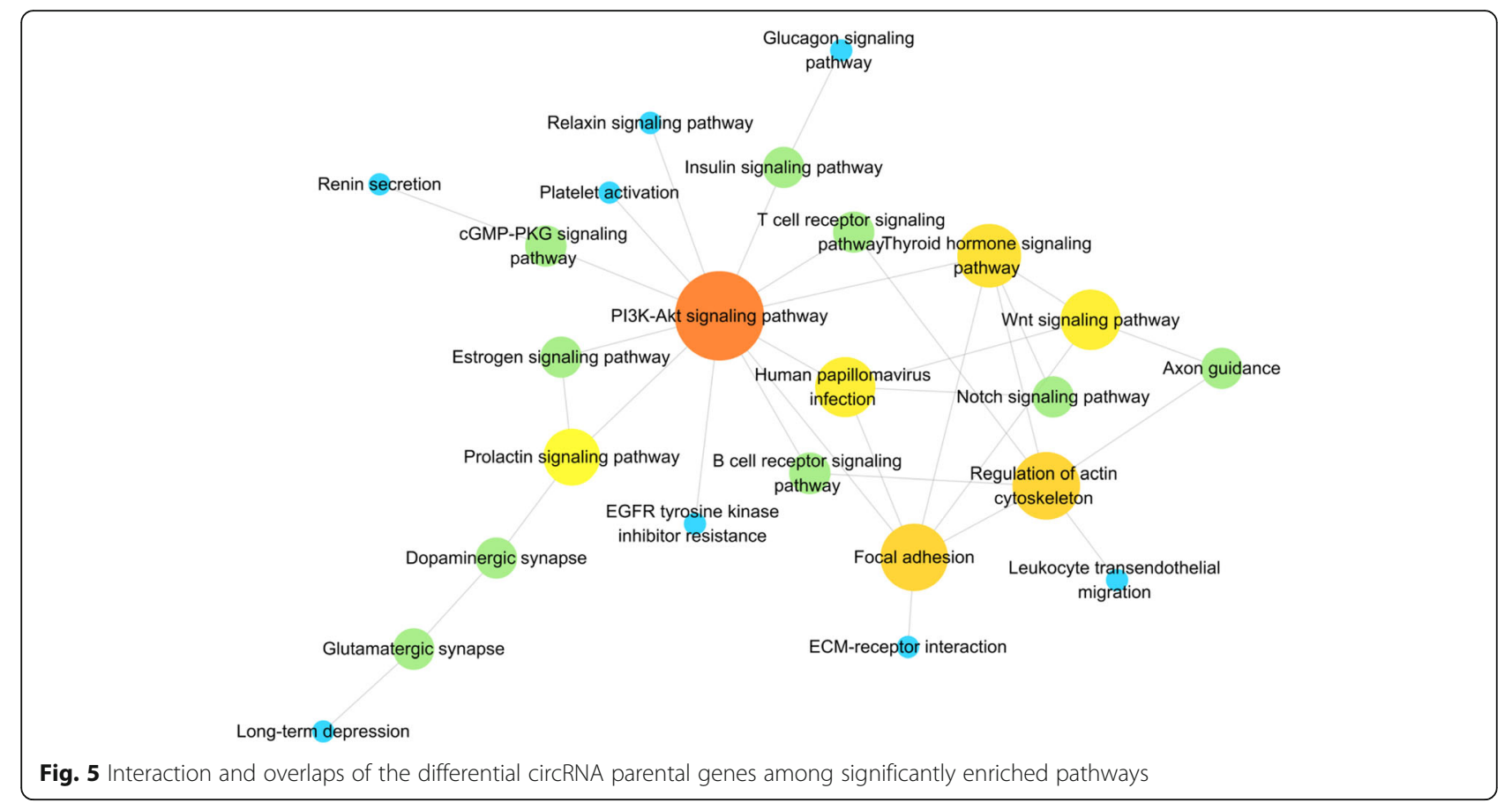

such as transcription and splicing regulation, miRNA sponges, mRNA traps, translational modulation, and post-translational modification [22]. The parental genes of circ_0077260, circ_0090100 and circ_0076206 are CGA, SAT1 and MTCH1, respectively. Of these parental genes, CGA encodes for the common alpha subunit of four glycoprotein hormones, hCG (human chorionic gonadotropin), LH (luteinizing hormone), FSH (folliclestimulating hormone) and TSH (thyroid-stimulating hormone) [23]. Previous studies have found that $\alpha$-hCG is correlated with PE [24], and CGA was differentially expressed in placenta tissue among late-onset $\mathrm{PE}$, earlyonset PE and healthy controls [25, 26]. CGA is also considered as a novel estrogen receptor response gene in breast cancer and an outstanding candidate marker for predicting response to endocrine therapy [27]. Further studies are needed to determine whether the association among circ_0077260, CGA and estrogen is involved in the pathogenesis of $\mathrm{PE}$.

GO and KEGG pathway analyses were performed to predict the biological functions and potential pathways of these differential circRNAs. Notablely, several pathways were found to be significantly enriched, such as focal adhesion, glycosaminoglycan degradation, fatty acid metabolism, fatty acid biosynthesis and Notch signaling pathway. It is well known that focal adhesion is crucial to trigger cell adhesion and many other cellular processes including cell migration, spreading and proliferation [28], which are important in PE development. And localization studies in placental tissues have showed that cytotrophoblasts in all stages of differentiation express focal adhesion kinase [29]. In terms of metabolic process, PE has been demonstrated to be associated with increased insulin resistance, hypertriglyceridemia, high circulating free fatty acids, low high-density lipoprotein particles, and high maternal and fetal plasma amino acid concentrations [30]. These metabolic alterations may contribute to the pathophysiology of the syndrome and may also influence fetal growth. For Notch signaling pathway, defects in this pathway would have adverse effect on placentation. And it has been suggested that Notch pathway down-regulation is associated with $\mathrm{PE}$ [31]. Further constructed pathway network showed that the exchanges with these pathways largely depended on the existence of PI3K-Akt signaling pathway. The PI3KAkt signaling pathway has been demonstrated to be a critical pathway mediating the growth-factor-dependent regulation of trophoblast growth and invasion [32]. The insufficient invasion of trophoblasts is known to be correlated with the development of PE [32]. Together, the altered circRNAs are associated with metabolic process, trophoblast growth and invasion related signaling pathways. Efficient biomarkers underlying these pathways need to be further investigated.

A large amount of evidence have indicated that exosomal circRNAs could act as ceRNA molecules or efficient miRNA sponges to regulate miRNA-targeted gene expression, transcription and protein synthesis [33-35]. The circRNAs may have many miRNA binding sites that competitively bind to miRNAs, and then alleviate the inhibitory effects of miRNAs on target molecules [21]. In this study, through circRNA/miRNA interactions 
analysis, we found that most of the exosomal circRNAs had miRNA binding sites, and some miRNAs were associated with PE. For example, miR-17-3p, miR-197, miR$424, \mathrm{miR}-431$ and miR-483 were reported to be aberrantly expressed in preeclamptic placenta [36-40]. miR17-3p and miR-424-5p were matched with circ_0077260, which was verified to upregulated in the umbilical cord blood exosomes of PE patients; miR-197-5p and miR431-5p potentially binds to downregulated circ_0076206; whereas miR-424-5p and miR-483-3p potentially matched with upregulated circ_0090100. Specifically, exosomal miR-486-1-5p and miR-486-2-5p were reported to be upregulated in PE pregnancy compared with normal pregnancy [41]. And miR-486-5p was matched with downregulated circ_0076206. In addtion, miR-885-5p was increased in plasma from PE patients compared with healthy pregnant women, and it was released into circulation mainly inside exosomes [42], whereas miR-885-5p potentially matched with upregulated circ_0077260. Therefore, we hypothesized that the role of exosomal circRNAs in PE development may be related to miRNA-mediated effects. The potential mechanism of the circRNA-miRNA-target gene interaction in $\mathrm{PE}$ is worthy of further study.

\section{Conclusions and prospect}

Our study firstly showed that exosomal circRNAs are aberrantly expressed in the umbilical cord blood of PE patients. The potential roles of these differentially expressed circRNAs and their interactions with miRNAs were further predicted through bioinformatics analysis, highlighting the importance of exosomal circRNAs in the pathogenesis of $\mathrm{PE}$ and providing a basis for further studies on function and mechanism of exosomal circRNAs in PE development. Studying the structure of circRNAs may lead to the development of effective artificial sponges to regulate the progression of disease. Artificial miRNA sponge technology, as an effective and stable miRNA inhibitor, may become a new direction of RNA gene therapy. It inhibits the expression of other miRNAs at the same time and produces a more persistent inhibition. In addition, because the expression profile of exosomal circRNAs in PE patients is different from that in the healthy group, combinations of exosomes and circRNAs are beneficial for their clinical application as diagnostic and prognostic biomarkers. Larger cohort studies are warranted to demonstrate that exosomal circRNAs are clinically applicable biomarkers.

\section{Abbreviations}

CircRNAs: Circular RNAs; PE: Preeclampsia; GO: Gene Ontology; KEGG: Kyoto Encyclopedia of Genes and Genomes; QPCR: Quantitative real-time PCR; GAPDH: Glyceraldehyde phosphate dehydrogenase; MREs: miRNA response elements; SD: Standard deviation; FC: Fold change; ceRNA: Competing endogenous RNA; hCG: Human chorionic gonadotropin; LH: Luteinizing hormone; FSH: Follicle-stimulating hormone; TSH: Thyroid-stimulating hormone

\section{Supplementary Information}

The online version contains supplementary material available at https://doi. org/10.1186/s12884-021-03777-7

Additional file 1: Table S1 Clinical data for the GDM patients and normal controls. Table S2 GO enrichment analysis of the circRNA parental genes. Table S3 KEGG enrichment analysis of the circRNA parental genes. Table S4 Prediction of circRNA/microRNA interactions

\section{Acknowledgements}

Not applicable.

\section{Authors' contributions}

$M C$ conceived and designed the idea, did data collection, wrote and drafted the manuscript. JW, $C B$ and $C L$ did data collection. $Y L$ did literature review. $\mathrm{HZ}$ and $\mathrm{YG}$ reviewed the manuscript. ZS performed the data analysis. $\mathrm{YZ}, \mathrm{WL}$ and $L Z$ designed, contributed to the reviewing of the final manuscript. All authors approved the final format of the submitted manuscript.

\section{Funding}

This work was supported in part by Jiangsu Provincial Key Research and Development Program (BE2020626), the National Natural Science Foundation of China (82071672), Six talent peaks project in Jiangsu Province (WSN-121, YY-112), Wuxi Key Medical Disciplines (ZDXK12), Jiangsu Maternal and Child Health Research Project (F201827, F201852), Top Talent Support Program for Young and Middle-Aged People of Wuxi Health Committee (BJ2020076, BJ2020089), Wuxi Science and Technology Development Fund (N20192039, N20201006, N20202003) and Project of Wuxi Health Commission (M202003). These funders had no any role in design of the study, data collection, analysis, interpretation of the data and preparation of the manuscript.

\section{Availability of data and materials}

The raw data of microarray in this study has been uploaded to GEO repository (GEO accession number is GSE166846, https://www.ncbi.nlm.nih. gov/geo/query/acc.cgi?acc=GSE166846). And all datasets generated for this study are included in the manuscript and the supplementary files.

\section{Declarations}

Ethics approval and consent to participate

All subjects gave written informed consent. This study was approved by the ethics committee of Women's Hospital of Nanjing Medical University (NFKS L2018-107), and all methods were carried out from September 2019 until October 2020, in accordance with the 1964 Principles of the Helsinki

Declaration and its later amendments.

Consent for publication

Not applicable.

Competing interests

The authors declare that they have no competing interests.

\section{Author details}

${ }^{1}$ Department of Obstetrics, The Affiliated Wuxi Maternity and Child Health Care Hospital of Nanjing Medical University, Wuxi 214002, China. ${ }^{2}$ Nanjing Maternity and Child Health Care Institute, Women's Hospital of Nanjing Medical University, Nanjing Maternity and Child Health Care Hospital, Nanjing 210004, China. ${ }^{3}$ Research Institute for Reproductive Medicine and Genetic Diseases, The Affiliated Wuxi Maternity and Child Health Care Hospital of Nanjing Medical University, Wuxi 214002, China. ${ }^{4}$ Department of Obstetrics, Women's Hospital of Nanjing Medical University, Nanjing Maternity and Child Health Care Hospital, Nanjing 210004, China. ${ }^{5}$ Department of Neonatology, The Affiliated Wuxi Children's Hospital of Nanjing Medical University, Wuxi 214023, China. 
Received: 18 January 2021 Accepted: 5 April 2021

\section{Published online: 15 April 2021}

\section{References}

1. Mol BWJ, Roberts CT, Thangaratinam S, Magee LA, de Groot CJM, Hofmeyr GJ. Pre-eclampsia. Lancet. 2016;387(10022):999-1011. https://doi.org/10.101 6/S0140-6736(15)00070-7.

2. Redman CW, Sargent IL. Latest advances in understanding preeclampsia. Science. 2005;308(5728):1592-4. https://doi.org/10.1126/science.1111726.

3. American College of $\mathrm{O}$, Gynecologists, Task Force on Hypertension in $\mathrm{P}$. Hypertension in pregnancy. Report of the American College of Obstetricians and Gynecologists' Task Force on Hypertension in Pregnancy. Obstet Gynecol. 2013;122(5):1122-31.

4. Ananth CV, Keyes KM, Wapner RJ. Pre-eclampsia rates in the United States, 1980-2010: age-period-cohort analysis. Bmj. 2013;347(nov07 15):f6564. https://doi.org/10.1136/bmj.f6564.

5. Valdiviezo C, Garovic VD, Ouyang P. Preeclampsia and hypertensive disease in pregnancy: their contributions to cardiovascular risk. Clin Cardiol. 2012; 35(3):160-5. https://doi.org/10.1002/clc.21965.

6. Bokslag A, van Weissenbruch M, Mol BW, de Groot CJ. Preeclampsia; short and long-term consequences for mother and neonate. Early Hum Dev. 2016;102:47-50. https://doi.org/10.1016/j.earlhumdev.2016.09.007.

7. Davis EF, Lazdam M, Lewandowski AJ, Worton SA, Kelly B, Kenworthy Y, et al. Cardiovascular risk factors in children and young adults born to preeclamptic pregnancies: a systematic review. Pediatrics. 2012;129(6): e1552-61. https://doi.org/10.1542/peds.2011-3093.

8. Hessvik NP, Llorente A. Current knowledge on exosome biogenesis and release. Cell Mol Life Sci. 2018;75(2):193-208. https://doi.org/10.1007/s00018017-2595-9.

9. Gurunathan S, Kang MH, Jeyaraj M, Qasim M, Kim JH. Review of the Isolation, Characterization, Biological Function, and Multifarious Therapeutic Approaches of Exosomes. Cells. 2019;8(4):307. https://doi.org/10.3390/cells8040307.

10. Pillay P, Moodley K, Moodley J, Mackraj I. Placenta-derived exosomes: potential biomarkers of preeclampsia. Int J Nanomedicine. 2017;12:8009-23. https://doi.org/10.2147/IJN.S142732.

11. Jayabalan N, Lai A, Ormazabal V, Adam S, Guanzon D, Palma C, et al. Adipose tissue Exosomal proteomic profile reveals a role on placenta glucose metabolism in gestational diabetes mellitus. J Clin Endocrinol Metab. 2019;104(5):1735-52. https://doi.org/10.1210/jc.2018-01599.

12. Salomon C, Scholz-Romero K, Sarker S, Sweeney E, Kobayashi M, Correa P, et al. Gestational diabetes mellitus is associated with changes in the concentration and bioactivity of placenta-derived Exosomes in maternal circulation across gestation. Diabetes. 2016;65(3):598-609. https://doi.org/1 0.2337/db15-0966.

13. Mitchell MD, Peiris HN, Kobayashi M, Koh YQ, Duncombe G, Illanes SE, et al. Placental exosomes in normal and complicated pregnancy. Am J Obstet Gynecol. 2015;213(4 Suppl):S173-81. https://doi.org/10.1016/j.ajog.2015.07.001.

14. Li Y, Zheng Q, Bao C, Li S, Guo W, Zhao J, et al. Circular RNA is enriched and stable in exosomes: a promising biomarker for cancer diagnosis. Cell Res. 2015;25(8):981-4. https://doi.org/10.1038/cr.2015.82.

15. Wang Y, Liu J, Ma J, Sun T, Zhou Q, Wang W, et al. Exosomal circRNAs: biogenesis, effect and application in human diseases. Mol Cancer. 2019; 18(1):116. https://doi.org/10.1186/s12943-019-1041-z.

16. Dai X, Chen C, Yang Q, Xue J, Chen X, Sun B, et al. Exosomal circRNA_ 100284 from arsenite-transformed cells, via microRNA-217 regulation of $\mathrm{EZH} 2$, is involved in the malignant transformation of human hepatic cells by accelerating the cell cycle and promoting cell proliferation. Cell Death Dis. 2018;9(5):454. https://doi.org/10.1038/s41419-018-0485-1.

17. Li J, Li Z, Jiang P, Peng M, Zhang $X$, Chen $K$, et al. Circular RNA IARS (circ-IARS) secreted by pancreatic cancer cells and located within exosomes regulates endothelial monolayer permeability to promote tumor metastasis. J Exp Clin Cancer Res. 2018;37(1):177. https://doi.org/10.1186/s13046-018-0822-3.

18. Csorba R, Yilmaz A, Tsikouras P, Wieg C, Teichmann A, von Tempelhoff GF. Rheological parameters in the umbilical cord blood in moderate and severe forms of preeclampsia. Clin Hemorheol Microcirc. 2013;55(4):391-401. https://doi.org/10.3233/CH-131776.

19. Kwon JY, Maeng YS, Kwon YG, Kim YH, Kang MH, Park YW. Decreased endothelial progenitor cells in umbilical cord blood in severe preeclampsia. Gynecol Obstet Investig. 2007;64(2):103-8. https://doi.org/10.1159/000100081.

20. Yusuf K, Kamaluddeen M, Hasan SU, Al-Awad E, Finch RA, Akierman AR. lonized calcium levels in umbilical cord blood of women with preeclampsia and normotensive pregnancies. J Maternal-fetal Neonat Med. 2012;25(2): 203-5. https://doi.org/10.3109/14767058.2011.561895.

21. Hansen TB, Jensen TI, Clausen BH, Bramsen JB, Finsen B, Damgaard CK, et al. Natural RNA circles function as efficient microRNA sponges. Nature. 2013; 495(7441):384-8. https://doi.org/10.1038/nature11993.

22. Shao T, Pan YH, Xiong XD. Circular RNA: an important player with multiple facets to regulate its parental gene expression. Mol Ther Nucleic Acids. 2021;23:369-76. https://doi.org/10.1016/j.omtn.2020.11.008.

23. Bieche I, Parfait B, Nogues C, Andrieu C, Vidaud D, Spyratos F, et al. The CGA gene as new predictor of the response to endocrine therapy in $E R$ alpha-positive postmenopausal breast cancer patients. Oncogene. 2001; 20(47):6955-9. https://doi.org/10.1038/sj.onc.1204739.

24. Moodley D, Moodley J, Buck R, Haneef R, Payne A. Free alpha-subunits of human chorionic gonadotropin in preeclampsia. Int J Gynaecol Obstet. 1995;49(3):283-7. https://doi.org/10.1016/0020-7292(95)02375-M.

25. Liu J, Song G, Meng T, Zhao G. Identification of differentially expressed genes and signaling pathways in placenta tissue of early-onset and lateonset pre-Eclamptic pregnancies by integrated bioinformatics analysis. Med Sci Monitor. 2020;26:e921997.

26. Varkonyi T, Nagy B, Fule T, Tarca AL, Karaszi K, Schonleber J, Hupuczi P, Mihalik N, Kovalszky I, Rigo J, Jr. et al: Microarray profiling reveals that placental transcriptomes of early-onset HELLP syndrome and preeclampsia are similar. Placenta 2011, 32 Suppl:S21-S29, DOI: https://doi.org/10.1016/.jplacenta.2010.04.014.

27. Bieche I, Parfait B, Le Doussal V, Olivi M, Rio MC, Lidereau R, et al. Identification of CGA as a novel estrogen receptor-responsive gene in breast cancer: an outstanding candidate marker to predict the response to endocrine therapy. Cancer Res. 2001;61(4):1652-8.

28. Zhu L, Plow EF, Qin J. Initiation of focal adhesion assembly by Talin and kindlin: a dynamic view. Protein Sci. 2020.

29. Ilic D, Genbacev O, Jin F, Caceres E, Almeida EA, Bellingard-Dubouchaud V, et al. Plasma membrane-associated pY397FAK is a marker of cytotrophoblast invasion in vivo and in vitro. Am J Pathol. 2001;159(1):93108. https://doi.org/10.1016/S0002-9440(10)61677-X.

30. von Versen-Hoeynck FM, Powers RW. Maternal-fetal metabolism in normal pregnancy and preeclampsia. Front Biosci. 2007;12(1):2457-70. https://doi. org/10.2741/2247.

31. Fragkiadaki P, Soulitzis N, Sifakis S, Koutroulakis D, Gourvas V, Vrachnis N, et al. Downregulation of notch signaling pathway in late preterm and term placentas from pregnancies complicated by preeclampsia. PLoS One. 2015; 10(5):e0126163. https://doi.org/10.1371/journal.pone.0126163.

32. Zhu X, Cao Q, Li X, Wang Z. Knockdown of TACC3 inhibits trophoblast cell migration and invasion through the PI3K/Akt signaling pathway. Mol Med Rep. 2016;14(4):3437-42. https://doi.org/10.3892/mmr.2016.5659.

33. Li Z, Yanfang W, Li J, Jiang P, Peng T, Chen K, et al. Tumor-released exosomal circular RNA PDE8A promotes invasive growth via the miR-338/ MACC1/MET pathway in pancreatic cancer. Cancer Lett. 2018;432:237-50. https://doi.org/10.1016/j.canlet.2018.04.035.

34. Zhang H, Zhu L, Bai M, Liu Y, Zhan Y, Deng T, et al. Exosomal circRNA derived from gastric tumor promotes white adipose browning by targeting the miR-133/PRDM16 pathway. Int J Cancer. 2019;144(10):2501-15. https:// doi.org/10.1002/ijc.31977.

35. Xue J, Liu Y, Luo F, Lu X, Xu H, Liu X, et al. Circ100284, via miR-217 regulation of $E Z H 2$, is involved in the arsenite-accelerated cell cycle of human keratinocytes in carcinogenesis. Biochim Biophys Acta Mol Basis Dis. 2017;1863(3):753-63. https://doi.org/10.1016/j.bbadis.2016.12.018.

36. Han L, Luo QQ, Peng MG, Zhang Y, Zhu XH. miR-483 is downregulated in pre-eclampsia via targeting insulin-like growth factor 1 (IGF1) and regulates the PI3K/Akt/mTOR pathway of endothelial progenitor cells. J Obstet Gynaecol Res. 2021;47(1):63-72. https://doi.org/10.1111/jog.14412.

37. Tang $Q$, Gui J, Wu X, Wu W. Downregulation of miR-424 in placenta is associated with severe preeclampsia. Pregnancy Hyperten. 2019;17:109-12. https://doi.org/10.1016/j.preghy.2019.05.017.

38. Yang $X$, Meng T. MicroRNA-431 affects trophoblast migration and invasion by targeting ZEB1 in preeclampsia. Gene. 2019;683:225-32. https://doi.org/1 0.1016/j.gene.2018.10.015.

39. Xu P, Zhao Y, Liu M, Wang Y, Wang H, Li YX, et al. Variations of microRNAs in human placentas and plasma from preeclamptic pregnancy. Hypertension. 2014;63(6):1276-84. https://doi.org/10.1161/HYPERTENSIONAHA.113.02647.

40. Choi SY, Yun J, Lee OJ, Han HS, Yeo MK, Lee MA, et al. MicroRNA expression profiles in placenta with severe preeclampsia using a PNA-based microarray. Placenta. 2013;34(9):799-804. https://doi.org/10.1016/j.placenta.2013.06.006. 
41. Salomon C, Guanzon D, Scholz-Romero K, Longo S, Correa P, Illanes SE, et al. Placental Exosomes as early biomarker of preeclampsia: potential role of Exosomal MicroRNAs across gestation. J Clin Endocrinol Metab. 2017; 102(9):3182-94. https://doi.org/10.1210/jc.2017-00672.

42. Sandrim VC, Luizon MR, Palei AC, Tanus-Santos JE, Cavalli RC. Circulating microRNA expression profiles in pre-eclampsia: evidence of increased miR885-5p levels. BJOG. 2016;123(13):2120-8. https://doi.org/10.1111/1471-052 8.13903 .

\section{Publisher's Note}

Springer Nature remains neutral with regard to jurisdictional claims in published maps and institutional affiliations.

Ready to submit your research? Choose BMC and benefit from:

- fast, convenient online submission

- thorough peer review by experienced researchers in your field

- rapid publication on acceptance

- support for research data, including large and complex data types

- gold Open Access which fosters wider collaboration and increased citations

- maximum visibility for your research: over $100 \mathrm{M}$ website views per year

At $\mathrm{BMC}$, research is always in progress.

Learn more biomedcentral.com/submissions 